\title{
Design and Realization of Regional Power Grid Fault Diagnosis and Restoration System
}

\author{
Feng Shuo and Ma Haifeng
}

\begin{abstract}
With the scale of power grid being enlarged, the structure is more complex, the possibility of failure also showed a trend of growth, it made that the fault equipment can be promptly and accurately diagnosed after the fault and require a fast search for optimal recovery path, thus crying for the development of fault diagnosis and recovery system for the safety of power grid. A fault diagnosis and recovery system is designed and realized in this paper which will provide help for scheduling personnel by monitoring module, fault diagnosis, fault recovery and human-computer interaction. After the fault, the system can diagnosis the fault devices and provide the optimal recovery path for the unfaulted area. The proposed system has been put into operation and proved to be valid.
\end{abstract}

Index Terms-Power grid, monitor, fault diagnosis, fault recovery, human-computer interaction.

\section{INTRODUCTION}

With modern grid scale being enlarged and the structure and operating conditions being more and more complex, the requirement of customers for power quality is increasing rapidly and the allowed blackout interval is getting smaller. It made that the grid can promptly and accurately identify the fault zone and diagnosis the fault equipment after the fault, quickly find the optimal recovery path and achieve the fault restoration efficiently and effectively. Especially in the smart grid environment, self-healing is a basic characteristic of it. Therefore what must be solved for power grid operation is how to put power grid fault diagnosis and recovery into use.

This paper discussed a fault diagnosis and recovery system on four aspects involving monitoring module, fault diagnosis, fault recovery and human-computer interaction. And it is of great significance and practical value for extending the function of the existing integration system for scheduling and control, improving the level of intelligence and realizing the power grid's intelligent alarm and recovery.

\section{FundAMENTAL FunCtion OF SYSTEM}

The fault diagnosis and recovery system for regional power grid is applied for rapid diagnosis of fault equipment, fault process reconstruction and the recovery issues. Based on the module architecture in Fig. 1, the specific function of the system is described below.

1) To realize fault diagnosis using real-time fault information based on the integration system for

Manuscript received September 9, 2013; revised October 27, 2013.

The authors are with Power Operation and Maintenance Department of Hegang Power Supply Company (e-mail: weijuanba@163.com). scheduling and control and to get real-time fault identification and accurately diagnosis of the fault equipment [1].

2) A fault process reconstruction based on the fault diagnosis for scheduling personnel to accurately grasp the fault events [2].

3) Analysis of the fault devices and fault zones with that of the power flow and the power system security and stability, providing operational recovery strategies for scheduling personnel and managers and achieving an efficient and reasonable fault restoration [3].

4) The simulation of various fault can provide emergency exercises for scheduling personnel to improve the ability to deal with the emergency.

5) Seeking scientific, prompt and accurate means to handle the emergency in regional power grid.

\section{The Overall Program Design}

The module architecture of the fault diagnosis and recovery system is showed in Fig. 1. It consists of monitoring module, fault diagnosis, fault recovery and human-computer interaction.

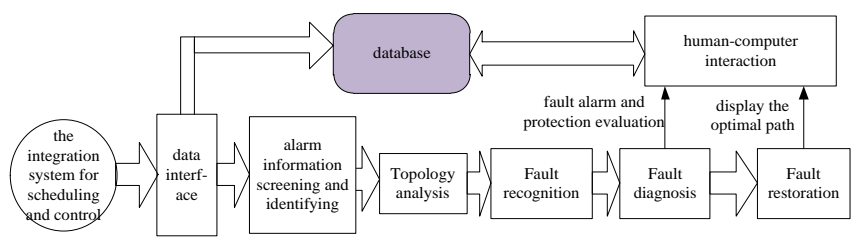

Fig. 1. The module architecture of the fault diagnosis and recovery system.

\section{A. Monitoring Module}

It includes data interface, alarm information screening and identifying, Topology analysis and fault recognition.

1) Data interface

Though building data interface with CC2000 system, the system designed here import the CIM model to get the grid equipment model and its connections, then parse them. And using the graphic data information in SVG graphics files, station wiring diagrams can be displayed on the human-computer interaction through the drawing module in the system. And real-time operating data information of grid can be getting through the network programming.

Also, data can be received and transmitted through the data interface. Furthermore, we can get fault information and response scheme when fault occurs.

2) Alarm information screening and identifying

When fault occurs a large number of signal will appear in 
the alarm screen, most of which is accompanied by uploading signal and cannot directly response the fault, thus seriously interfering the judgment for fault.

The information [4] can be screened and synthesized in this module, and repeated and shaking information can be deleted.

\section{3) Topology analysis}

Dynamic connection of the equipment and analysis charged are Provided. Electrical equipment are topology colored according to their voltage level and uncharged equipment are displayed in gray.

\section{4) Fault Recognition}

Failure criterion has been made through which we can judge whether there is a fault and prevent the daemon of fault diagnosis and recovery from getting started by mistake.

\section{B. Fault Diagnosis}

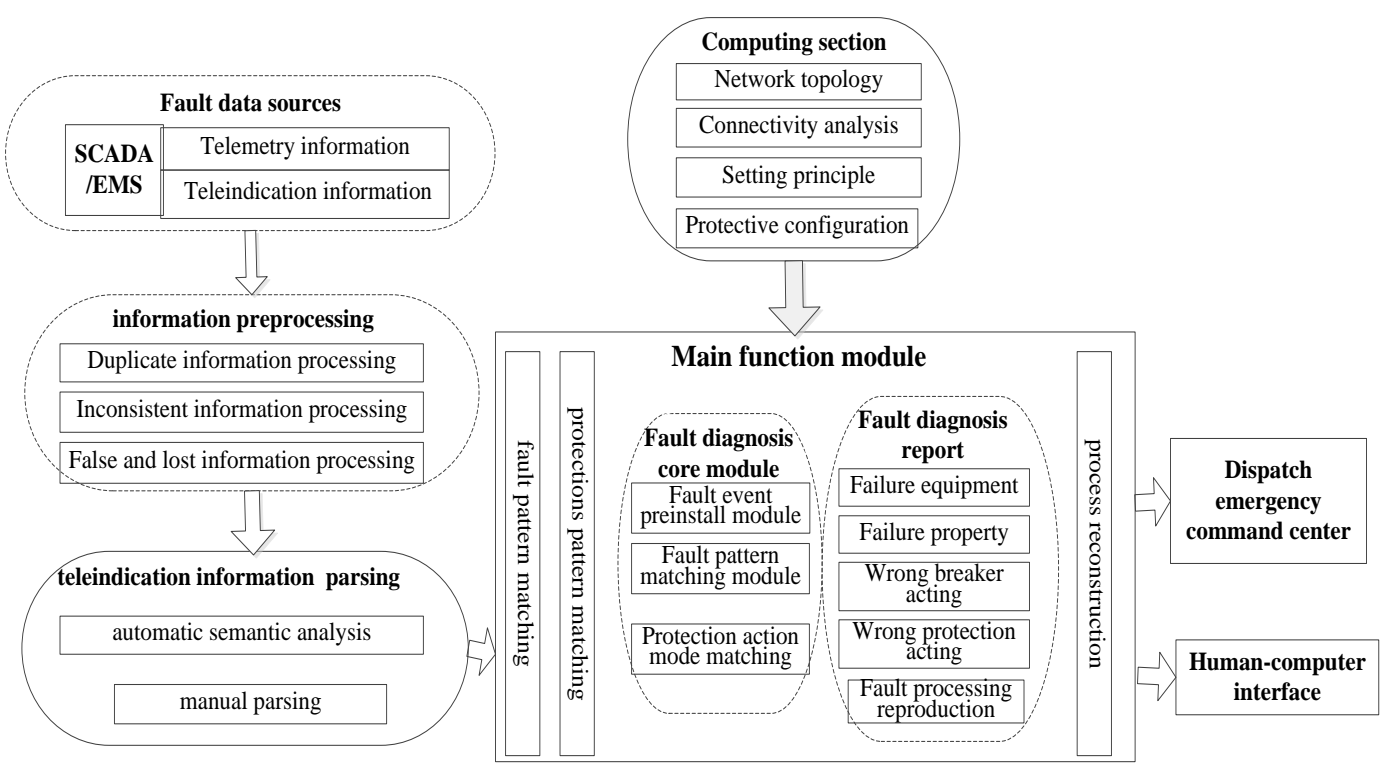

Fig. 2. The fault diagnosis system diagram.

Fault diagnosis is using all kinds of fault data to analyze fault with a variety of methods [5], [6], to get an accurate diagnosis of fault equipment and evaluate the switch action and protection action, and finally to provide a diagnostic report. The diagnosis system diagram is showed as Fig. 2.

\section{1) Information processing}

As the fault information collected is incomplete and inaccurate, information processing becomes necessary before fault diagnosis. The processing contains duplicate information processing, inconsistent information processing and the missending and lost information processing. The data gets valid for fault diagnosis system after the processing.

\section{2) Tele-Indication information parsing}

The tele-indication information got though data interface with CC2000 system has to be modeling analysed and translated to specific data. The modeling analysis includes automatic semantic analysis and manual parsing. The automatic semantic analysis can parse tele-indication information to get data about substations, electrical equipment, objects like whether it's primary protection or backup protection and state data. When it turns out that a piece of tele-indication information cannot get automatically parsed or its analytical result is partially missing, the analytical result has to be manually improved according to parameter database.

\section{3) Fault diagnosis}

Fault diagnosis here is studied based on protection configuration principle, the characteristics power grid structure's change under the fault and relevant cases. This system can give an accurate diagnosis for a simple fault without mistakenly acting elements and a result based on probability analysis for a complicated fault with wrong action.

Fault diagnose contains fault pattern matching, protections pattern matching and process reconstruction. The fault patterns mainly include fault isolation of a single failure and regional fault patterns etc.

After the fault diagnosis, the result can be delivered as diagnostic reports and displayed in the human-computer interaction interface.

\section{4) Historical fault query}

The fault cases can be searched according to basic search criteria like fault occurrence time, electrical equipment and protective action information.

- Choose a period of time and all faults occurred in this period can be queried;

- Faults can be queried according to protective action information. And inversely protective information can be queried based on failure events and collected and saved. An historical library can be got for archiving and selecting which failure to report.

\section{Fault Restoration}

Fault restoration is studied with comprehensive consideration of multiple factors like network topology, load flow calculation, contingency plans for regional accidents and personnel operating frequency in recovery path and the analogy analysis of the operation in fault zone [7], [8].

As the Fig. 3 shows, the fault restoration module consists of the main function module, computation module and the 
restricting module. The main function of the restoration module includes fault isolation, network reconstruction, island recovery, special recovery for fault and the forming of the operation tickets.

\section{1) Fault isolation}

Once fault occurs, obtain the specific diagnosis information from the diagnosis system and isolate the fault devices promptly

\section{2) Network reconstruction}

Make an overload analysis for areas without blackout. If overload, it is necessary to do network reconstruction and corresponding overload treatment scheme will be given to eliminate overload.

\section{3) Island recovery}

For the unfaulted outage subsystem, search all paths, and it's necessary to evaluate the out-of-limit of flow and voltage, network loss and the number of switching operations to select the optimal path. The final recovery solution can be displayed in the human-computer interaction interface.

In order to provide reference for dispatchers, not only the recovery path can be showed dynamically through the interface, but also the evaluation parameters of all paths. Furthermore, dispatchers can adjust the evaluation parameters for actual needs.

\section{4) Special recovery for fault}

As the accident recovery solutions and brownout schemes and other related recovery strategy in various regional scheduling are different, a special handling module is set to handle the load and accidents with special demands.

\section{5) Operation tickets}

Deliver operation tickets after the optimal path has been selected. The operation tickets mainly include device information in blackout area, fault device information, the switch operation information and recovery path which is shown as operational sequence.

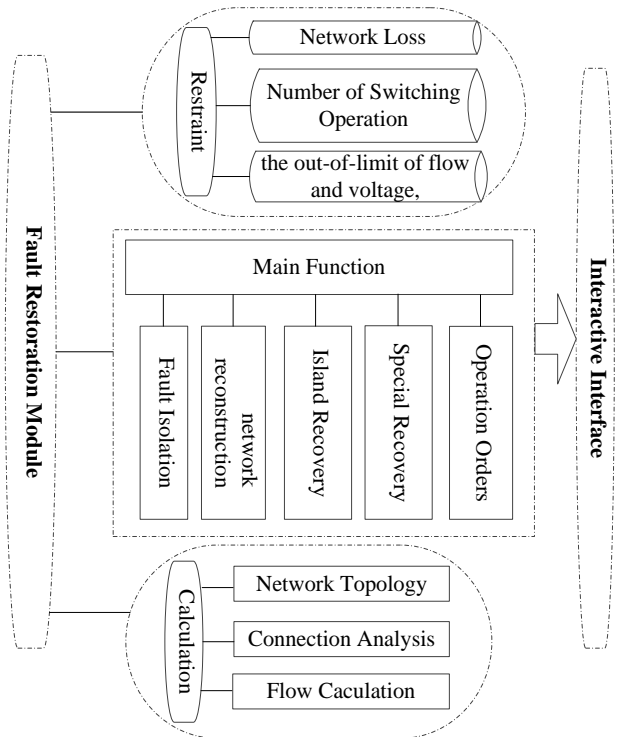

Fig. 3. Functional diagram of restoration module.

\section{Human-Computer Interaction}

1) The connection diagrams of regional power grid are displayed in the human-computer interaction interface normally.

2) Once a fault occurs, real-time alarm is triggered and fault areas are marked. The recovery path can be showed dynamically through the interface.

3) Also, the fault process can be repeated in the interface.

\section{Software Testing}

The fault diagnosis and recovery system designed here is easily realized and convenient and have a friendly and intuitive interface and small amount of maintenance. Due to the low probability of grid fault, fault simulation can be conducted in the software through the human-computer interaction interface to test its effectiveness and practicality. Reading the alarm information from the database and setting fault condition to test the software, the results show that the software running smoothly and can give a correct and reasonable result.

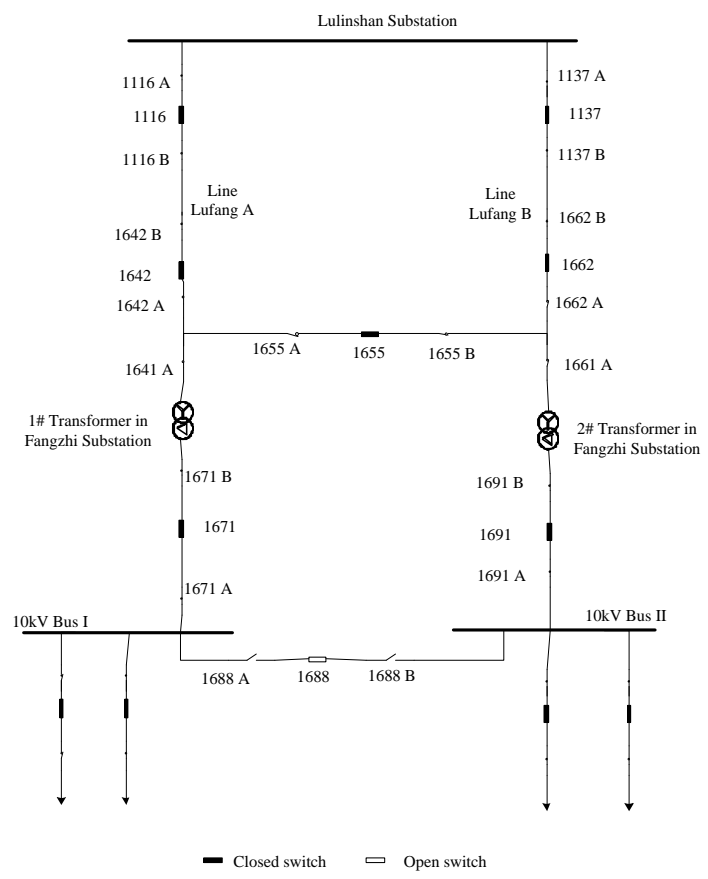

Fig. 4. The regional grid map.

Test the system under the failure of the main transformer II in $110 \mathrm{kV}$ Fangzhi Substation. The normal operation before the fault is shown in Fig. 4.

Set the fault through simulating the displacement information of switches on both ends of transformer II and differential protection action signal of II; As shown in Fig. 4, the diagnosis module gets simulation information, give an accurate diagnosis promptly and display the suspected fault device in the graphical interface and give an alarm. Then deliver a diagnose report and set up the recovery module which will get operation tickets through steps like network topology and load flow calculation and so on. The details are as Table I shows.

In addition, after the fault, the interface switches to Fangzhi Substation and the failure device transformer II is marked, the optimal path can be shown colored red dynamically in the interface, as shown in the Fig. 5 shows. 
TABLE I: THE DIAGNOSE REPORT

\begin{tabular}{|c|c|c|}
\hline \multicolumn{3}{|r|}{ DATE: 2013/ 2/ 18} \\
\hline \multicolumn{2}{|c|}{ Operation Task } & Isolate Transformer in Fangzhi Substation \\
\hline Sequence & $\begin{array}{l}\text { Operation } \\
\text { Unit }\end{array}$ & Operation Procedure \\
\hline 1 & Fangzhi & Switch off the disconnector 1691B \\
\hline 2 & Fangzhi & Switch off the disconnector $1691 \mathrm{~A}$ \\
\hline \multicolumn{2}{|c|}{ Operation Task } & $\begin{array}{l}\text { Restore electricity for 10kV Bus in Fangzhi } \\
\text { Substation }\end{array}$ \\
\hline Sequence & $\begin{array}{c}\text { Operation } \\
\text { Unit }\end{array}$ & Operation Procedure \\
\hline 1 & Fangzhi & Switch on the disconnector $1688 \mathrm{~A}$ \\
\hline 2 & Fangzhi & Switch on the disconnector $1688 \mathrm{~B}$ \\
\hline 3 & Fangzhi & Switch on the breaker 1688 \\
\hline \multicolumn{2}{|c|}{ Operation Task } & Energize $110 \mathrm{kV}$ Lufang Line B \\
\hline Sequence & $\begin{array}{c}\text { Operation } \\
\text { Unit }\end{array}$ & Operation Procedure \\
\hline 1 & Lulinshan & Switch on the breaker 1137 \\
\hline 2 & Fangzhi & Switch on the breaker 1162 \\
\hline 3 & Fangzhi & Switch on the inner bridge breaker 1655 \\
\hline
\end{tabular}

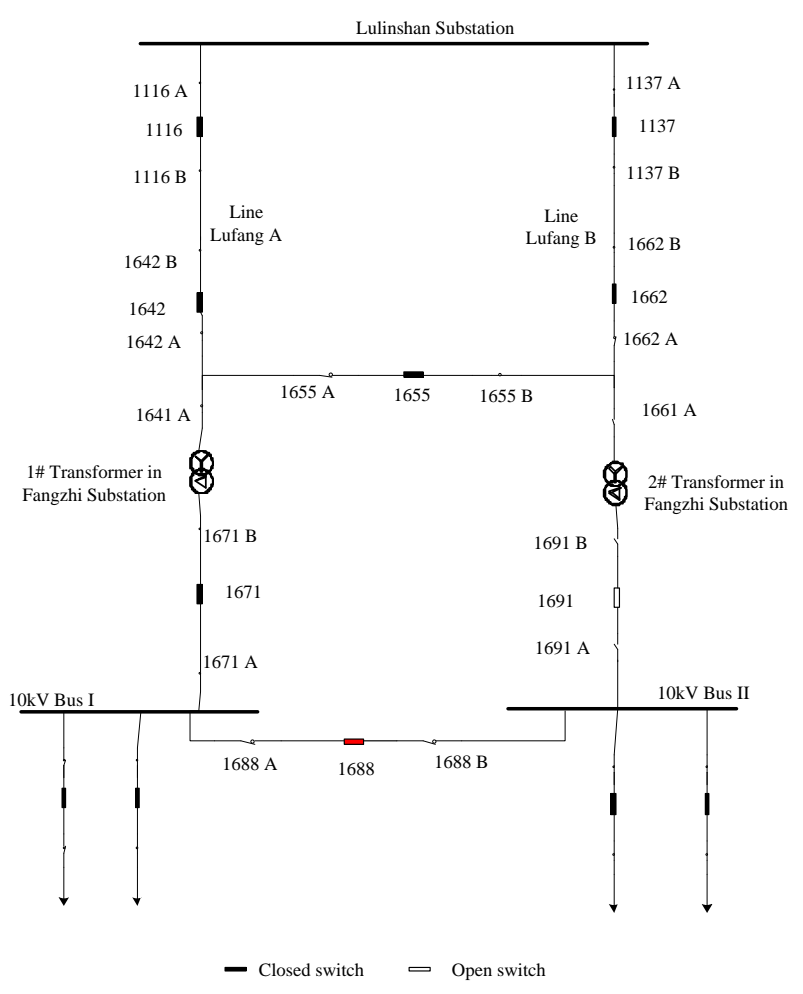

Fig. 5. The recovery path shown dynamically.

\section{CONCLUSION}

The fault diagnosis and recovery system designed in this paper can realize an accurate identification of the fault equipment after the fault, a fast recovery of power supply in unfaulted outage subsystem. The operation tickets will be got and the fault equipment and final recovery solution will be displayed in the human-computer interaction interface. It can be convenient operated and provide help for scheduling personnel to treat the grid fault emergency, thus being of great significance for improving the security and reliability of the power system operation.

\section{REFERENCES}

[1] X. Xu, J. Zhang, and S. M. Sun, "Assistant decision-making system for intelligent dispatching in regional power grid," Automation of Electric Power Systems, vol. 4, pp.111-115, Jun. 2012.

[2] J. Ding, X. M. Bai, and W. Zhao, "Fault information analysis and diagnosis method of power system based on complex event processing technology," in Proc. the CSEE, vol. 27, 2007.

[3] D. B. Liu, X. P. Gu, and J. Q. Zhao, "Practical research of fault restoration for the regional power grid," Power System Protection and Control, vol. 38, 2010.

[4] M. Kezunovic, C. W. Fromen, and D. R. Sevcik, "An expert system for transmission substation event analysis," IEEE Trans. on Power Delivery, vol. 8, pp.1942-1949, 1993.

[5] J. D. Xu et al., "Dual data source based fault diagnosis application of the regional power network," Automation of Electric Power Systems, vol. 30, pp. 68-72, 2006

[6] W. H. Chen, "Online fault diagnosis for power transmission networks using fuzzy digraph models," IEEE Trans. on Power Delivery, vol. 27, pp. $688-698,2012$

[7] M. R. Kleinberg, K. Miu, and H. D. Chiang, "Improving service restoration of power distribution systems through load curtailment of in-service customers," IEEE Trans. Power Systems, vol. 26, pp. 1110-1117, 2011.

[8] M. H. Liu, D. Y. Zhang, and P. Y. Zou, "Regional network fault restoration system applied in on-line mode," Power System Technology, vol. 30, pp. 35-39, Sep. 2006.

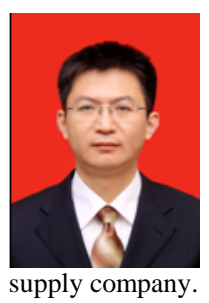

Feng Shuo was born in August 4, 1971 in Shandong province and graduated from the major of power plant and power system in Shenyang electric power college in 1992. He engaged in safety in production work in Hegang power supply company, and has worked as the substation attendant and the director of substation running work area. Now he is the director of power operation and maintenance department of Hegang power

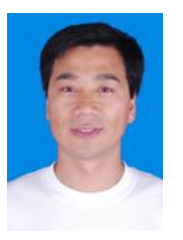

Ma Haifeng was born in May 2, 1971, and now he is the vice director of power operation and maintenance department of Hegang power supply company . 